\title{
EWSR1-BEND2 fusion defines an epigenetically distinct subtype of astroblastoma
}

\author{
Calixto-Hope G. Lucas ${ }^{1} \cdot$ Rohit Gupta ${ }^{1}$. Jasper $\mathrm{Wu}^{1} \cdot$ Kathan Shah $^{1} \cdot$ Ajay Ravindranathan ${ }^{1}$. Jairo Barreto ${ }^{1}$. \\ Melissa Gener ${ }^{2} \cdot$ Kevin F. Ginn ${ }^{3}$ O Owen W. J. Prall ${ }^{4} \cdot$ Huiling Xu$^{4} \cdot$ Damien Kee $^{5} \cdot$ Hyun S. Ko $^{6} \cdot$ Nausheen Yaqoob $^{7}$. \\ Nida Zia ${ }^{8} \cdot$ Adriana Florez $^{9} \cdot$ Soonmee Cha ${ }^{10}$. Arie Perry ${ }^{1,11}$. Jennifer L. Clarke ${ }^{12,13}$. Susan M. Chang ${ }^{12}$. \\ Mitchel S. Berger ${ }^{11} \cdot$ David A. Solomon ${ }^{1}$ (1)
}

Received: 14 October 2021 / Revised: 5 November 2021 / Accepted: 17 November 2021 / Published online: 25 November 2021

(c) The Author(s) 2021

Astroblastoma is a glial neoplasm previously diagnosed solely on the basis of histologic features including tumor cells with stout processes arranged in perivascular pseudorosettes (so-called "astroblastomatous" rosettes), often embedded within a sclerotic stroma [2, 3]. However, refinement in molecular-based tumor classification schemes has led to the realization that this histologic pattern is not specific to astroblastoma and can also be seen in other genetically defined tumor entities, including $B R A F$-mutant pleomorphic xanthoastrocytoma, ZFTA-fused supratentorial ependymoma, and $I D H$-wildtype glioblastoma [1, 7, 13]. A recent genomics study demonstrated that a large subset of tumors with astroblastoma histologic appearance harbor $M N 1$ gene fusion (typically with BEND2 as the fusion partner) and resolve into a distinct epigenetic cluster, which was initially termed "high-grade neuroepithelial tumor, MN1-altered" [11]. Multiple subsequent studies have confirmed that MN1

David A. Solomon

david.solomon@ucsf.edu

1 Department of Pathology, University of California, San Francisco, 513 Parnassus Ave, Health Sciences West 451, San Francisco, CA 94143, USA

2 Department of Pathology, Children's Mercy Hospital, Kansas City, MO, USA

3 Department of Pediatric Hematology and Oncology, Children's Mercy Hospital, Kansas City, MO, USA

4 Department of Pathology, Peter MacCallum Cancer Centre, University of Melbourne, Melbourne, VIC, Australia

5 Department of Medical Oncology, Peter MacCallum Cancer Centre, University of Melbourne, Melbourne, VIC, Australia

6 Department of Cancer Imaging, Peter MacCallum Cancer Centre, University of Melbourne, Melbourne, VIC, Australia

7 Department of Histopathology, Indus Hospital and Health Network, Karachi, Pakistan fusions define a group of circumscribed glial neoplasms with astroblastoma-like morphology (with or without a primitive embryonal-like component), female predominance, and a propensity for local recurrence, but with mostly favorable clinical outcomes [1, 7, 13]. The identification of $M N 1$ fusion by next-generation sequencing or MN1 rearrangement by break-apart fluorescent in situ hybridization (FISH) can be used to support the diagnosis of "astroblastoma, MN1altered" per recent cIMPACT-NOW recommendations [8], and this molecularly-defined tumor type has been adopted in the $5^{\text {th }}$ edition of the WHO Classification of Tumors of the Central Nervous System [4]. Of note, however, are a few recent case reports of gliomas with astroblastoma-like histology lacking MN1 alterations and instead harboring EWSR1$B E N D 2$ fusion [9, 10, 12, 14]. The biologic relationship of these gliomas with EWSR1-BEND2 fusion compared to the

8 Department of Pediatric Hematology and Oncology, Indus Hospital and Health Network, Karachi, Pakistan

9 Department of Pathology, Fundación Santafé de Bogotá, Bogota, Colombia

10 Department of Radiology and Biomedical Imaging, University of California, San Francisco, San Francisco, CA, USA

11 Department of Neurological Surgery, University of California, San Francisco, San Francisco, CA, USA

12 Division of Neuro-Oncology, Department of Neurological Surgery, University of California, San Francisco, San Francisco, CA, USA

13 Department of Neurology, University of California, San Francisco, San Francisco, CA, USA 
a

\begin{tabular}{|c|c|c|l|l|l|l|l|l|}
\hline Pt \# & $\begin{array}{c}\text { Age at dx } \\
\text { (yrs) }\end{array}$ & Sex & $\begin{array}{l}\text { Clinical } \\
\text { presentation }\end{array}$ & Location & $\begin{array}{l}\text { Histologic } \\
\text { features }\end{array}$ & Fusion & Methylation class & Source \\
\hline 1 & 20 & $\mathrm{M}$ & Arm weakness & Brainstem & Astroblastoma & EWSR1-BEND2 & Astroblastoma, EWSR1-BEND2 fused & current study \\
\hline 2 & 6 & $\mathrm{~F}$ & Arm weakness & Spinal cord & Astroblastoma & EWSR1-BEND2 & Astroblastoma, EWSR1-BEND2 fused & current study \\
\hline 3 & 26 & $\mathrm{~F}$ & Seizure & Right frontal & Astroblastoma & EWSR1-BEND2 & Astroblastoma, EWSR1-BEND2 fused & Current study \\
\hline 4 & 6 & $\mathrm{~F}$ & Seizure & Left frontal & Astroblastoma & EWSR1-BEND2 & Astroblastoma, EWSR1-BEND2 fused & current study \\
\hline- & 38 & $\mathrm{M}$ & Leg numbness & Spinal cord & Astroblastoma & EWSR1-BEND2 & reported as 'HGNET, MN1' & Smith-Cohn et al \\
\hline- & 0 & $\mathrm{M}$ & Unknown & Spinal cord & Astroblastoma & EWSR1-BEND2 & reported as 'HGNET, MN1' & Yamasaki et al \\
\hline- & 36 & $\mathrm{M}$ & Leg numbness & Spinal cord & Astroblastoma & EWSR1-BEND2 & reported as 'HGNET, MN1' & Tsutsui et al \\
\hline- & Unknown & Unknown & Unknown & Unknown & Astroblastoma & EWSR1-BEND2 & Not performed & Ramkissoon et al \\
\hline
\end{tabular}
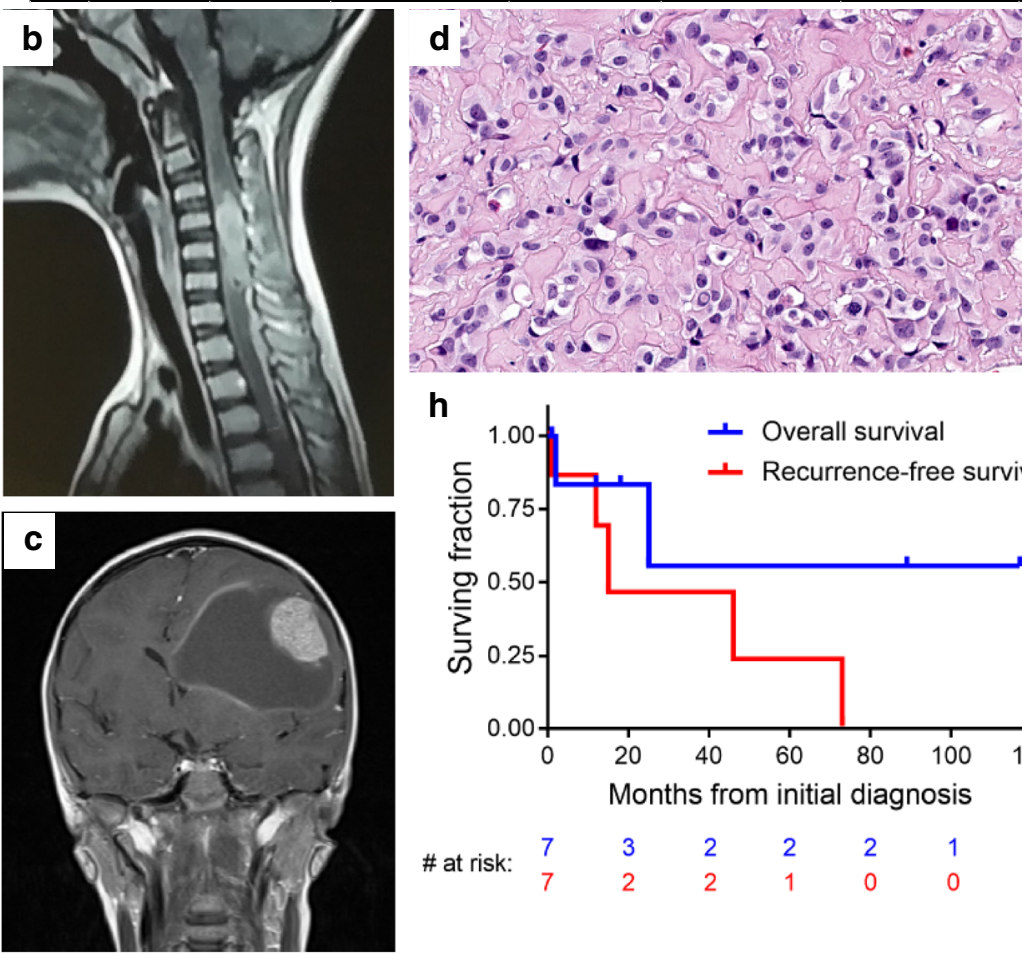

h

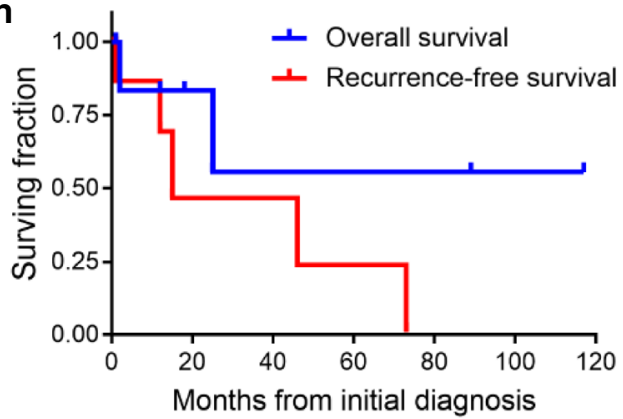

\# at risk: $\begin{array}{lllllll}7 & 3 & 2 & 2 & 2 & 1 & 0\end{array}$

i

Astroblastoma, MN1-altered

EWSR1-BEND2

fused gliomas

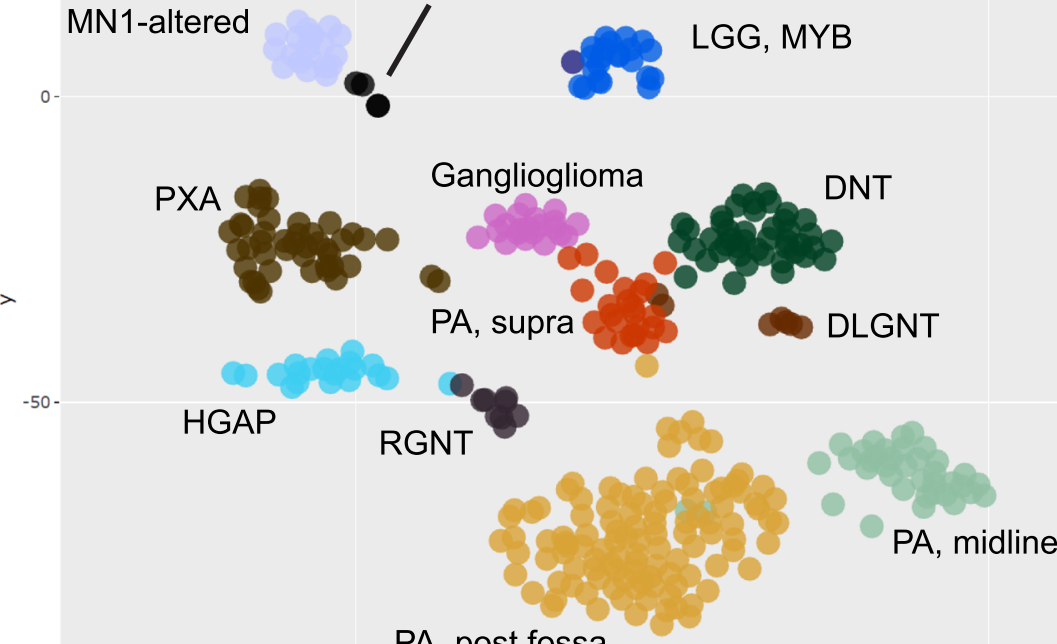

PA, post fossa

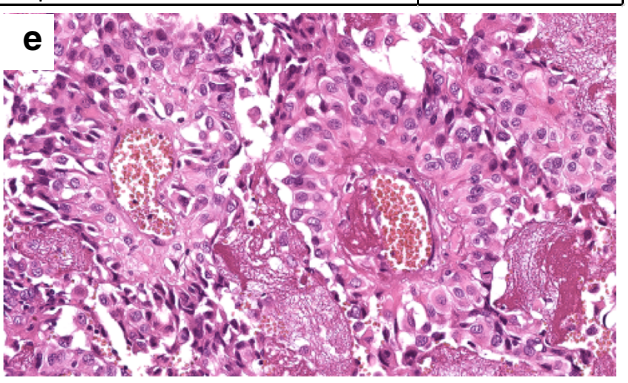

f

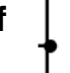

$\begin{array}{llllllll}0 & 10 & 20 & 30 & 40 & 50 & 60 & 70\end{array}$

Patient age (years)

g

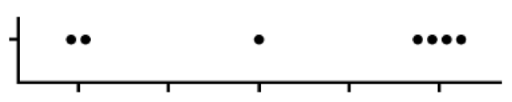


४Fig. 1 Clinicopathologic features and epigenomic profiling of astroblastoma-like gliomas with EWSR1-BEND2 fusion. a Summary table of the eight patients with gliomas harboring EWSR1-BEND2 fusion, four from this study and four previously reported. b MR imaging from patient \#2 demonstrating an enhancing mass within the cervical spinal cord. c MR imaging from patient \#4 demonstrating a solid and cystic mass with an enhancing mural nodule in the left frontal lobe of the brain. d Histology from patient \#1 showing a glial neoplasm with dense pericellular and perivascular sclerosis. e Histology from patient \#3 showing astroblastomatous rosettes. f Dot plot of patient age at diagnosis for astroblastoma-like gliomas with EWSR1-BEND2 fusion. $\mathbf{g}$ Dot plot of tumor anatomic location for astroblastoma-like gliomas with EWSR1-BEND2 fusion. h Kaplan-Meier plot of overall survival and recurrence-free survival for astroblastoma-like gliomas with EWSR1-BEND2 fusion. i tSNE dimensionality reduction plot of genome-wide DNA methylation profiles from the 4 EWSR1$B E N D 2$ fused gliomas alongside 366 reference tumors spanning 11 CNS tumor entities. $\mathbf{j}$ Unsupervised hierarchical clustering of DNA methylation data from the 4 astroblastoma-like gliomas with EWSR1BEND2 fusion and 3 astroblastomas with confirmed MN1-BEND2 fusion, with a heatmap of the 20,000 most differentially CpG sites shown

newly defined tumor type "astroblastoma, $M N 1$-altered" is currently unresolved.

We performed targeted next-generation sequencing and genome-wide DNA methylation profiling on a cohort of four patients with EWSR1-BEND2 fused gliomas. The one male and three females had a median age at time of initial diagnosis of 13 years (range 6-26 years) (Fig. 1a). Two patients had tumors located in the frontal lobe and presented with seizures, whereas the other two patients had tumors located in the brainstem or cervical spinal cord and presented with limb weakness. Imaging revealed enhancing mass lesions, sometimes associated with a cystic cavity (Fig. 1b, c; Supplementary Fig. 1 [Online Resource 1]). Extent of resection, adjuvant therapy, and clinical outcomes are listed in Supplementary Table 1 [Online Resource 2]. Histologically, these were circumscribed gliomas composed of epithelioid tumor cells embedded within a densely sclerotic stroma, with variably prominent astroblastomatous rosettes (Fig. 1d, e; Supplementary Fig. 2 [Online Resource 1]; Supplementary Table 2 [Online Resource 2]). A more primitive, embryonal-like component was prominent in two tumors and focally present in the other two. Mitotic activity was conspicuous (often $>5$ mitoses per $2 \mathrm{~mm}^{2}$ ), and foci of necrosis were identified in all cases, but were not associated with peripheral palisading of tumor cells. No microvascular proliferation, Rosenthal fibers, or eosinophilic granular bodies were identified. Immunohistochemical evaluation revealed variable patchy expression of glial markers (GFAP and OLIG2), minimal to absent expression of neuronal markers (synaptophysin and neurofilament), and often diffuse membranous expression of EMA (Supplementary Fig. 3 [Online Resource 1]; Supplementary Table 3 [Online Resource 2]). Notably, immunostaining for BCOR demonstrated diffuse strong nuclear positivity in two tumors, despite an absence of $B C O R$ mutation, tandem duplication, or other gene rearrangement. Meta-analysis of patient outcomes and other clinical data together with previous case reports revealed occurrence during childhood or early adulthood, location most frequently in midline structures (5/7 in the brainstem or spinal cord), and a propensity for local recurrence, with CSF dissemination occurring in two patients leading to mortality (Fig. 1f-h) [9, 10, 12, 14].

By targeted next-generation DNA sequencing, all four tumors demonstrated EWSR1 fusion with BEND2 as the 3' partner (Supplementary Fig. 4 [Online Resource 1]; Supplementary Table 4 [Online Resource 2]). In three of the tumors, the gene fusion linked exons 1-8 (codons 1-270) of EWSR1 with exons 2-14 (codons 9-799) of BEND2, whereas the remaining tumor linked exons 1-7 (codons 1-199) of EWSR1 with exons 4-14 (codons 126-799) of BEND2. The EWSR1-BEND2 fusion was the solitary oncogenic alteration identified in all tumors, with an absence of accompanying alterations involving $I D H 1 / 2$, histone $\mathrm{H} 3$ genes, $B R A F, N F 1$, PRKCA, FGFR1/2/3, NTRK1/2/3, EGFR, PDGFRA, MET, PIK3CA, PIK3R1, PTEN, CDKN2A, TP53, TERT (including promoter region), ATRX, CIC, FUBPI, MN1, ZFTA (c11orf95), RELA, YAP1, MYC, MYCN, MYB, and MYBL1 [6]. The quantity of chromosomal copy number aberrations was variable, but no tumors harbored whole arm codeletion of chromosomes $1 \mathrm{p}$ and 19q, focal amplifications, or homozygous deletions (Supplementary Fig. 5 [Online Resource 1]; Supplementary Table 5 [Online Resource 2]). Genome-wide DNA methylation profiling was performed on the four tumors using Infinium EPIC 850k Beadchips (Illumina) following the manufacturer's recommended protocols (see Supplementary Methods [Online Resource 3]). tSNE clustering of the DNA methylation data alongside a reference cohort of CNS tumors revealed that the EWSR1$B E N D 2$ fused gliomas resolved into a single epigenetic group that was divergent from all established reference methylation classes of CNS tumors, but was in close proximity to "High-grade neuroepithelial tumor, $M N 1$-altered" (Fig. 1i; Supplementary Fig. 6 [Online Resource 1]; sample manifest in Supplementary Table 6 [Online Resource 2]). Random forest classification using the online DKFZ Brain Tumor Classifier (www.molecularneuropathology. org) demonstrated high-confidence match to the "HGNET, MN1" methylation class for all four tumors (Supplementary Table 7 [Online Resource 2]) [5]. Unsupervised hierarchical clustering successfully segregated the 4 EWSRI-BEND2 fused gliomas from 3 astroblastomas with confirmed $M N 1$ BEND2 fusion but revealed highly similar DNA methylation patterns overall (Fig. 1j).

Here we show that gliomas with EWSR1-BEND2 fusion exhibit astroblastoma-like histologic features and resolve into a single epigenetic subgroup most similar to the methylation class "HGNET, MN1" that encompasses the 
new tumor type astroblastoma, $M N 1$-altered. It remains unclear why MN1-BEND2 and EWSR1-BEND2 fusions are selected for in astroblastoma, and why EWSRl fusion appears to be more common in brainstem and spinal cord astroblastomas relative to $M N 1$ fusion in hemispheric tumors, but we speculate that these represent two biologically distinct subtypes of astroblastoma which awaits confirmation in future studies. In epigenetically-defined astroblastomas with available sequencing data delineating the fusion partners, only a single tumor with $M N 1$ fused to a partner other than BEND2 has been reported to date (dkfz_CNS-PNET_15-0184, GSM1881234 with MN1$C X X C 5$ fusion) [11]. Including this case series, there are now eight reported astroblastomas with EWSR1-BEND2 fusion (seven with available epigenetic data), indicating that BEND2 may be a more critical gene for molecular oncogenesis and relevant for tumor classification compared to $M N 1$. This is reminiscent of how supratentorial ependymomas were initially defined as being RELA fusion positive, but the recognition of a group of supratentorial ependymomas with ZFTA (C11orf95) fused to other partners besides RELA has led to a revised nosology based on ZFTA as the defining fusion partner. Thus, these findings support consideration of $B E N D 2$ fusion as the defining molecular alteration for astroblastoma, rather than $M N 1$ rearrangement as is currently recognized. Future studies are required to define the full biologic and clinicopathologic spectrum of astroblastomas, including establishment of evidence-based grading criteria and determination of optimal treatment regimens.

Supplementary Information The online version contains supplementary material available at https://doi.org/10.1007/s00401-021-02388-y.

Acknowledgements C.G.L. is supported by the UCSF Training Program in Translational Brain Tumor Research, National Cancer Institute, NIH (T32 CA151022) and the UCSF Brain Tumor SPORE Developmental Research Program Award from the National Cancer Institute, NIH (P50 CA097257). D.A.S. is supported by the NIH Director's Early Independence Award from the Office of the Director, National Institutes of Health (DP5 OD021403), the UCSF Brain Tumor SPORE Developmental Research Program Award from the National Cancer Institute, NIH (P50 CA097257), the Morgan Adams Foundation, the Yuvaan Tiwari Foundation, the UCSF Glioblastoma Precision Medicine Program, and the Panattoni Family Foundation. We thank the staff of the UCSF Clinical Cancer Genomics Laboratory for assistance with genetic profiling.

Data availability Digitally scanned image files of representative H\&E and immunostained sections from the 4 tumors are available at the following link: https://figshare.com/projects/Astroblastoma-like_gliom as_with_EWSR1-BEND2_fusion/124222. DNA methylation array data files are available from the Gene Expression Omnibus (GEO) repository under accession number GSE183972 (https://www.ncbi.nlm.nih. gov/geo/). Structural variant and copy number data are available in the electronic supplementary material. Raw sequencing data files are available upon request.

\section{Declarations}

Conflict of interest A.P. and D.A.S. are members of the editorial board for Acta Neuropathologica. They were not involved in the assessment or decision-making process for this manuscript. The other authors declare that they have no competing interests related to this report.

Ethical approval This study was approved by the Committee on Human Research of the University of California, San Francisco, with a waiver of patient consent.

Open Access This article is licensed under a Creative Commons Attribution 4.0 International License, which permits use, sharing, adaptation, distribution and reproduction in any medium or format, as long as you give appropriate credit to the original author(s) and the source, provide a link to the Creative Commons licence, and indicate if changes were made. The images or other third party material in this article are included in the article's Creative Commons licence, unless indicated otherwise in a credit line to the material. If material is not included in the article's Creative Commons licence and your intended use is not permitted by statutory regulation or exceeds the permitted use, you will need to obtain permission directly from the copyright holder. To view a copy of this licence, visit http://creativecommons.org/licenses/by/4.0/.

\section{References}

1. Boisseau W, Euskirchen P, Mokhtari K et al (2019) Molecular profiling reclassifies adult astroblastoma into known and clinically distinct tumor entities with frequent mitogen-activated protein kinase pathway alterations. Oncologist 24:1584-1592

2. Bonnin JM, Rubinstein LJ (1989) Astroblastomas: a pathological study of 23 tumors, with a postoperative follow-up in 13 patients. Neurosurgery 25:6-13

3. Brat DJ, Hirose Y, Cohen KJ, Feuerstein BG, Burger PC (2000) Astroblastoma: clinicopathologic features and chromosomal abnormalities defined by comparative genomic hybridization. Brain Pathol 10:342-352

4. WHO Classification of Tumours Editorial Board (2021) World Health Organization Classification of central nervous system tumours, 5th edn. International Agency for Research on Cancer, Lyon

5. Capper D, Jones DTW, Sill M et al (2018) DNA methylationbased classification of central nervous system tumours. Nature 555:469-474

6. Kline CN, Joseph NM, Grenert JP et al (2017) Targeted nextgeneration sequencing of pediatric neuro-oncology patients improves diagnosis, identifies pathogenic germline mutations, and directs targeted therapy. Neuro Oncol 19:699-709

7. Lehman NL, Usubalieva A, Lin T et al (2019) Genomic analysis demonstrates that histologically-defined astroblastomas are molecularly heterogeneous and that tumors with MN1 rearrangement exhibit the most favorable prognosis. Acta Neuropathol Commun 7:42

8. Louis DN, Wesseling P, Aldape K et al (2020) cIMPACT-NOW update 6: new entity and diagnostic principle recommendations of the cIMPACT-Utrecht meeting on future CNS tumor classification and grading. Brain Pathol 30:844-856

9. Ramkissoon SH, Bandopadhayay P, Hwang J et al (2017) Clinical targeted exome-based sequencing in combination with 
genome-wide copy number profiling: precision medicine analysis of 203 pediatric brain tumors. Neuro Oncol 19:986-996

10. Smith-Cohn MA, Abdullaev Z, Aldape KD et al (2021) Molecular clarification of brainstem astroblastoma with EWSR1BEND2 fusion in a 38-year-old man. Free Neuropathol [online ahead of print]

11. Sturm D, Orr BA, Toprak UH et al (2016) New brain tumor entities emerge from molecular classification of CNS-PNETs. Cell 164:1060-1072

12. Tsutsui T, Arakawa Y, Makino Y et al (2021) Spinal cord astroblastoma with EWSR1-BEND2 fusion classified as HGNETMN1 by methylation classification: a case report. Brain Tumor Pathol [online ahead of print]
13. Wood MD, Tihan T, Perry A et al (2018) Multimodal molecular analysis of astroblastoma enables reclassification of most cases into more specific molecular entities. Brain Pathol 28:192-202

14. Yamasaki K, Nakano Y, Nobusawa S et al (2020) Spinal cord astroblastoma with an EWSR1-BEND2 fusion classified as a high-grade neuroepithelial tumour with MN1 alteration. Neuropathol Appl Neurobiol 46:190-193

Publisher's Note Springer Nature remains neutral with regard to jurisdictional claims in published maps and institutional affiliations. 